\title{
Genre et textualité lyrique : l'impossible modélisation comme indice de transgénéricité
}

\section{Sandra Glatigny}

\section{(2) OpenEdition}

Journals

\section{Édition électronique}

URL : http://journals.openedition.org/pratiques/3737

DOI : 10.4000/pratiques.3737

ISSN : 2425-2042

\section{Éditeur}

Centre de recherche sur les médiations (CREM)

\section{Édition imprimée}

Date de publication : 1 juin 2013

Pagination : 105-118

\section{Référence électronique}

Sandra Glatigny, «Genre et textualité lyrique : l'impossible modélisation comme indice de transgénéricité », Pratiques [En ligne], 157-158| 2013, mis en ligne le 18 décembre 2017, consulté le 20 avril 2019. URL : http://journals.openedition.org/pratiques/3737 ; DOI : 10.4000/pratiques.3737

Ce document a été généré automatiquement le 20 avril 2019.

(c) Tous droits réservés 


\title{
Genre et textualité lyrique : l'impossible modélisation comme indice de transgénéricité
}

\author{
Sandra Glatigny
}

1 Cette réflexion est née de l'expérience anodine mais réitérée que voici : ouvrez n'importe quelle anthologie ou manuel scolaire et cherchez des textes lyriques. Vous vous trouverez alors face à un corpus varié qui s'étend de la « Ballade des pendus ${ }^{1}$ » de Villon à À la vieille Parque $^{2}$ de Jude Stéfan en passant par Les Contemplations ${ }^{3}$ de Victor Hugo. De fait, au cœur des traditions critiques, les termes «lyrisme», "lyrique» semblent appliqués indifféremment à des textes présentant pourtant des différences énonciatives, stylistiques et formelles extrêmement importantes. Face à cette disparité, le problème de la modélisation générique se pose de manière particulièrement prégnante. Quel modèle sélectionner pour définir le genre lyrique? Comment saisir l'originalité d'une écriture sans moyen de comparaison? Comment procéder, sinon de manière intuitive?

2 À partir de cette observation et au regard de cette hétérogénéité, il faudrait revoir le statut de genre du lyrisme. Contrairement au dramatique et à l'épique, il ne paraît pas être une catégorie issue de la convergence entre un modèle théorique et un ensemble de textes similaires. Le lyrisme semble échapper à un tel régime et s'inscrire davantage dans une logique transversale que dans une logique verticale. Le lyrisme ne permet pas ce double mouvement, inductif et déductif, des textes aux modèles et des modèles aux textes, qui fonde la classification générique. Après avoir rappelé les variations diachroniques subies par le genre, nous verrons que la confrontation entre modèles textuels et réflexions théoriques est dominée par l'écart et conduit à l'échec de la théorisation. Au terme de ce parcours, il faudra envisager la possibilité d'un autre fonctionnement, celui du lyrisme comme principe transgénérique. 


\section{Fluctuations diachroniques}

3 littéraire quelques jalons, représentatifs d'un genre. Or, pour ce qui concerne la textualité lyrique, il est délicat de dégager des textes emblématiques qui serviraient de repères pour les auteurs comme pour les lecteurs. Cette difficulté résulte d'abord de l'histoire du genre lyrique. En effet, ce dernier a subi une révolution presque complète : il est passé du statut de non genre, ou tout du moins de genre marginal, à celui d'hypergenre. reposait sur une longue histoire de justification et de réajustement des modèles platoniciens et aristotéliciens. Si l'épique et le dramatique sont restés relativement stables, la catégorie lyrique, réceptacle de toutes les formes littéraires marginales, est venue s'ajouter de manière artificielle. Malgré l'intégration à un système global, les critères pour définir le genre ont toujours été flous et variables et, par conséquent, les modèles disparates.

5 Genre circonstanciel et performanciel dans l'Antiquité, étroitement lié à la musique, le genre lyrique est valorisé par les poètes de la Renaissance. Dans sa préface des Odes, adressée au lecteur, Ronsard affirme s'être éloigné de ses contemporains, "prenant stile apart, sens apart, euvre apart » et « [s]'achemin[er] par un sentier inconnu, et monstrant le moien de suivre Pindare et Horace ${ }^{5}$ ». La contradiction manifeste entre imitation et innovation montre que l'on ne peut envisager la poésie lyrique au $\mathrm{XVI}^{\mathrm{e}}$ siècle comme une simple continuation des lyriques grecs et romains. Ce genre semble se prévaloir d'une tradition prestigieuse mais cette tradition se limite à des textes singuliers. Le lyrisme paraît constituer une sphère propice à la liberté et à l'innovation mais demeure soumis aux productions antérieures et aux exigences rhétoriques. Dans le même texte, Ronsard donne un autre élément définitoire: "c'est le vrai but d'un poète lyrique de célébrer jusques à l'extrémité celui qu'il entreprend de louer ${ }^{6} »$. Il inscrit donc le poème lyrique dans une logique épidictique. Mais, à considérer le corpus lyrique du $\mathrm{xvI}^{\mathrm{e}}$, cette orientation argumentative n'est pas constante. D'ailleurs, quelle unité trouver au sein même des Odes? Certains poèmes présentent une forme de communion collective et politique comme « l'Ode au Roi »:

Comme un qui prend une coupe, Seul honneur de son trésor, Et de rang verse à sa troupe, Du vin qui rit dedans l'or:

Ainsi versant la rousée,

Dont ma langue est arousée, Sus la race de Valois, En mon dous Nectar j'abreuve Le plus grand Roi qui se treuve, Soit en armes ou en lois. ${ }^{7}$

Ce type d'odes, épidictique et solennelle, s'oppose à d'autres, plus intimistes et plus personnelles, comme « Des baisers de Cassandre », « À Macée » ou « À Cassandre » :

Ma petite columbelle,

Ma petite toute belle,

Mon petit œil baisez moi :

D'un baiser qui lon tens dure, 
Poussez hors la peine dure

De mon amoureus esmoi. ${ }^{8}$ plus claire mais il n'en est rien. L'élaboration du système des genres s'effectue à partir des non-dits du texte aristotélicien. Les commentaires perpétuent ce silence: rien dans les Réflexions sur la poétique de Rapin ou si peu (il réduit le système des genres à deux pôles: représentation et narration), rien dans l'Art poétique de Boileau, si ce n'est des allusions indirectes grâce à une liste de formes données. Le lyrisme est rattaché au champ littéraire par défaut, uniquement par sa forme versifiée. La définition que l'on trouve dans le Dictionnaire de l'Académie de Furetière est éloquente. Elle relègue les vers lyriques au rang d'usurpateurs : «Les vers lyriques, sonnets, épigrammes et chansons ne méritent le nom de poème que fort abusivement ${ }^{9} \%$. Les énumérations que l'on trouvait déjà dans les traités poétiques de la Renaissance dessinent des ensembles de formes codifiées et proches de la musique mais en aucun cas un genre à part entière.

$\mathrm{Mu}$ par les réflexions des philosophes allemands et la valorisation progressive de la subjectivité, le système romantique résulte de cette longue histoire. À partir du XVIII ${ }^{\mathrm{e}}$ au sein du débat entre l'abbé Batteux et Schlegel notamment -, la notion de mimesis est élargie non plus à la simple représentation d'actions mais à la représentation des sentiments: la triade épique / dramatique / lyrique se met définitivement en place. Parallèlement, on assiste à une modification importante de la textualité lyrique. En témoignent les préfaces et textes liminaires des recueils du XIX : Lamartine ouvre la voie avec Les Méditations poétiques pour faire du genre lyrique le mode d'expression d'une vibration singulière et personnelle de l'âme. Le genre lyrique envahit la production littéraire et devient bientôt la forme dominante. Mais, au sein de cet ensemble, peut-on extraire un texte ou un recueil dont l'exemplarité nourrirait la textualité lyrique ? Doiton opter pour l'élégie lamartinienne, l'emphase hugolienne, le syncrétisme nervalien ou l'ironie baudelairienne? On le voit, la textualité lyrique se perd dans la multiplicité des modèles.

D'ailleurs, les excès sentimentaux du lyrisme romantique vont rapidement être battus en brèche et accentuer la confusion qui règne au sein du genre. Outre les parodies critiques telles qu'on les trouve chez Corbière ou Laforgue, des poètes comme Verlaine ou Rimbaud cultivent une poétique de mise à distance du " je ». Quant à Mallarmé, il fonde la théorie du genre sur l'objectivation du lyrisme, effaçant de l'énoncé les traces de sa profération. On ne peut donc plus s'appuyer sur la présence du sujet singulier et personnel pour délimiter le corpus. Ne reste de la lyrique romantique que le «développement d'une exclamation ${ }^{10} »$ suivant le propos de Paul Valéry.

11 Malgré cette brillante formule, définir le lyrisme moderne est tout aussi problématique, non seulement parce que la production qualifiée de lyrique est extrêmement variée, mais également parce que la singularité du lyrisme est totalement absorbée par le genre poétique. Dans Poésie et récit, Dominique Combe a montré que l'exclusion du récit du champ poétique (fin XIX-XX ) a amené à une bipartition générique : d'un côté, le récit et de l'autre la poésie confondue avec le lyrisme ${ }^{11}$. D'ailleurs, la plupart des ouvrages critiques contemporains, à leur corps défendant parfois, utilise les termes de poésie et de 
lyrisme comme s'ils étaient interchangeables. Dans le métatexte, lyrisme peut devenir synonyme de poésie.

$12 \mathrm{Au}$ terme de ce rapide rappel, on peut voir que le régime générique du lyrisme est profondément influencé par son évolution historique. On assiste à une complète révolution du genre passé de la marge à l'hypergenre. La conséquence est double. Lorsque le lyrisme se situe hors du système générique, les modèles textuels sont considérés comme inutiles parce qu'ils représentent un cadre secondaire, sans objet dans le champ littéraire. De fait, avant de constituer un genre à part entière, le lyrisme est resté marginal en raison de la hiérarchisation et de la dispersion des formes qui le constituaient. Si l'auteur et le lecteur élaborent des modèles, ces derniers restent à l'état de virtualité diffuse : l'atomisation de la textualité lyrique ne permet pas de construire un schéma de référence. Lorsqu'il devient hypergenre et recouvre l'ensemble de la production poétique, le lyrisme perd sa spécificité et, par conséquent, le modèle textuel est trop large pour en refléter les caractéristiques particulières. Il est donc délicat d'élaborer un modèle générique stable quand les discours théoriques comme leurs réalisations scripturales subissent des variations aussi importantes.

\section{Modèles et textes en confrontation : une relation conflictuelle}

13 À la multiplication des modèles dans le temps s'ajoute la multiplication de théories et de modèles, en décalage avec l'ensemble de la textualité lyrique. Les quelques approches théoriques suivantes montrent la relation conflictuelle entre les textes et leurs modèles, empêchant de fixer le statut générique du lyrisme. En effet, ce dernier semble signer la disjonction entre textes et métatextes, en raison notamment de la pluralité théorique qui s'y rattache. Dans les traités de poétique de la Renaissance, les poètes mélangent les approches formelles (formes codifiées et proximité avec la musique), thématiques et rhétoriques. Ceux de l'époque classique sont centrés sur la dimension morale. Les époques modernes sont plus axées sur des définitions anthropologiques, sociologiques ou philosophiques. Outre la disparité des critères définitoires, il est difficile de faire coïncider théorie, modèle et textes, sans déborder le champ littéraire.

D'un point de vue formel, le genre lyrique a souvent été réduit à des structures institutionnalisées comme l'ode ou le sonnet. Analysant les référents des noms de genre, J.-M. Schaeffer évoque les formes de la poésie lyrique, décrivant « une généricité fondée sur des conventions régulatrices ». Si l'on se réfère uniquement à ces normes, la poésie lyrique se résumerait à une « énumération de règles prescrites ${ }^{12}$ ». Mais, pour trouver une unité, ce critère n'est ni nécessaire, ni suffisant. En premier lieu, définir un genre par des sous genres ou par d'autres genres - suivant la classification choisie -, peut vite tourner à la tautologie. En second lieu, les formes fixes traditionnellement considérées comme lyriques sont extrêmement variées. Le mouvement épidictique de l'ode, poème lyrique par excellence selon Hugo (Odes et Ballades), la concision du sonnet ou l'universalité douloureuse de l'élégie composent un ensemble hétérogène. Ces formes, qui n'ont de fixes que le nom - puisque la plupart ont perdu leurs contraintes formelles -, comportent de multiples variations tonales et thématiques. Les formes fixes oscillent entre contraintes et déconstructions. Enfin, les innovations modernes, la déstructuration des formes fixes et l'intrusion du lyrisme dans des sphères qui lui sont étrangères, ne permettent pas de 
s'appuyer sur la seule logique formelle. La reproduction est loin d'être la règle. Certes, Nerval, Baudelaire ou Hugo s'inspirent de formes pérennisées par Ronsard ou Du Bellay. Mais sur quelles règles antérieures s'appuieraient des poèmes comme ceux de Senghor dans Chants d'ombre ${ }^{13}$, ou ceux d'Aragon dans Cantiques à Elsa?

Figure $^{14}$

\begin{tabular}{|c|c|c|c|}
\hline Cette valse & est un vin & qui ressemble & au Saumur \\
\hline Cette valse & est le vin & que j'ai bu & dans tes bras \\
\hline Tes cheveux & en sont l'or & et mes vers & s'en émurent \\
\hline \multicolumn{4}{|c|}{ Valsons-la comme on saute un mur } \\
\hline \multicolumn{2}{|c|}{ Ton nom s'y murmure } & Elsa valse & et valsera \\
\hline Lajeunesse & $y$ pétille & où nos jours & étant courts \\
\hline \multicolumn{2}{|c|}{ A Montmartre on allait } & oublier & qu'on pleura \\
\hline Notre nuit & a perdu & ce secret & du faux-jour \\
\hline \multicolumn{2}{|c|}{ Mais a-t-elle } & oubliél'amo & \\
\hline L'amour est & lourd & Elsa valse & et valsera $[$. \\
\hline
\end{tabular}

En l'occurrence, l'écriture lyrique est fondée sur la quête d'une rencontre intersémiotique entre rythme musical et représentation figurale de la valse. La recherche formelle y est essentielle mais la transgression est telle qu'elle signe la disparition du modèle du cantique et qu'elle dépasse le seul recours aux lettres. La constitution d'un corpus lyrique reposant sur la forme entraîne donc sa dispersion.

L'approche thématique parait tout aussi vaine. Par exemple, au XVIII ${ }^{\mathrm{e}}$, dans les Beaux arts réduits à un même principe, "sur la poésie lyrique ", l'abbé Batteux évoque tour à tour l'ode sacrée, héroïque, philosophique et anacréontique en les distinguant par leurs thèmes : la première traite de Dieu et de la foi, la deuxième de la gloire des héros, la troisième des vertus morales et la dernière du plaisir. Cette énumération est unifiée artificiellement par l'idée d'imitation des sentiments. Cependant, on voit bien qu'il n'y a pas de restriction dans les sujets abordés et que ceux-ci rencontrent les autres genres.

Parmi les définitions qui ont marqué le genre lyrique, on peut citer celle du philosophe Hegel. Dans son Esthétique, il écrit: "Ce qu'elle exprime, c'est le subjectif, le monde intérieur, les sentiments, les contemplations et les émotions de l'âme ; au lieu de retracer le développement d'une action, son essence et son but final sont l'expression des mouvements intérieurs de l'âme de l'individu. ${ }^{15}$ » Bien qu'Hegel soit un penseur de l'universalité, la notion de subjectivité a conduit lecteurs amateurs et avertis à voir dans la poésie lyrique, une poésie personnelle, autarcique et statique. Or, la production contemporaine et postérieure à Hegel vient démentir cette vision restrictive et cette interprétation erronée. Pour ce qui est de la dimension personnelle, la lecture des recueils du XIX ${ }^{e}$ siècle est édifiante. De l'ambition totalisatrice à la quête d'universalité des grands romantiques, nombreux sont les poètes qui ont recouru notamment au mythe pour faire de leurs passions singulières l'expression de l'humaine souffrance : «Quand je vous parle de vous, je vous parle de moi. Comment ne le sentez-vous pas? Ah! Insensé qui crois que je ne suis pas toi ${ }^{16} "$, affirme Victor Hugo au seuil des Contemplations.

Nietzsche, quant à lui, remet en cause la singularité et l'identification de la subjectivité du poète lyrique: «Le "je" du lyrique résonne donc du plus profond abîme de l'Être; sa 
"subjectivité", au sens des esthéticiens modernes, est pure illusion ${ }^{17}$ » Il voit dans le "je" lyrique une sorte d'absolu de l'être. La question du sujet lyrique a d'ailleurs largement occupé les philosophes et a rejailli dans l'analyse littéraire: sujet en devenir, sujet en crise, fictionnalisation du sujet, « $4^{\mathrm{e}}$ personne du singulier » pour reprendre l'expression de Jean-Michel Maulpoix ${ }^{18}$. Elle ne trouve jamais de réponse définitive. Quoiqu'il en soit, l'opposition subjectivité / objectivité paraît peu opératoire. La poésie lyrique a tendu à s'objectiver et à opérer la "disparition élocutoire du poète ${ }^{19}$ ». À partir de la deuxième moitié du XIXe, l'expression émotionnelle passe par la mise à distance du sujet et la dépersonnalisation de l'énoncé. Or, le retrait de la subjectivité entrave la capacité d'identification du texte lyrique pour le lecteur, quand bien même le «je " perdure. Certains poètes modernes en font même le thème de leur poème. C'est le cas d'Eugène Guillevic dans « Art poétique » :

Je ne parle pas pour moi,

Je ne parle pas en mon nom,

Ce n'est pas de moi qu'il s'agit.

Je ne suis rien

Qu'un peu de vie, beaucoup d'orgueil.

Je parle pour tout ce qui est,

$\mathrm{Au}$ nom de ce qui a forme et pas de forme.

Il s'agit de tout ce qui pèse,

De tout ce qui n'a pas de poids..$^{20}$

19 Le mouvement d'extériorisation émotionnelle entraîne la dissolution du « je » dans un « tout » sans limites. Plus largement, la manipulation ludique du sujet constitue l'un des moteurs de la poésie lyrique mais elle relève davantage de l'analyse psychanalytique et philosophique que de l'analyse linguistique.

Passons sur l'absence d'actions et par conséquent d'une progression, propre à la narration : doit-on exclure du corpus lyrique des poèmes comme «Elle était déchaussée, elle était décoiffée » d'Hugo ou "À une passante » de Baudelaire sous prétexte que ces textes mettent en scène des actions ? Dans ce dernier exemple, le récit elliptique prépare et assure l'efficacité de la charge lyrique qui culmine dans le dernier tercet:

Ailleurs, bien loin d'ici ! trop tard ! jamais peut-être !

Car j'ignore où tu fuis, tu ne sais où je vais,

Ô toi que j'eusse aimée, ô toi qui le savais ! ${ }^{21}$

21 Si le lyrisme ne raconte pas, à l'instar de l'épique, on peut en revanche reconnaître qu'il n'est pas exempt d'une dynamique que l'on peut souvent qualifier de narrative, bien qu'avortée. Ces auteurs recourent à des saynètes pour exprimer leurs sentiments intimes et personnels. Il s'agit pour le poète de donner une image de son intériorité et celle-ci ne peut éviter une mise en scène plus ou moins dynamique : paysage de l'âme de Verlaine, mythologie et nostalgie nervaliennes, projections fantasmatiques d'Aloysius Bertrand. La poésie lyrique ne se distingue pas de la poésie épique par la dynamique narrative. C'est son utilisation qui diffère. Elle est médiation de l'intériorité dans le premier cas, objet direct de la représentation dans le second. La transmission des sentiments s'effectue grâce à l'image et à la figuration, dépassant les cadres littéraires et artistiques pour recourir à d'autres moyens d'expression.

Les théories énonciatives découlent indirectement de ces réflexions romantiques sur le sujet. Dans Logique des genres littéraires, Käte Hamburger oppose le genre fictionnel au genre lyrique. Son approche philosophique, phénoménologique et linguistique l'amène à définir le texte lyrique comme un « énoncé de réalité » qui met en place un « complexe de 
sens ${ }^{22} »$. Aussi convaincante et stimulante que soit la définition du complexe de sens lyrique comme mouvement centripète, qui déporte la relation sujet/objet dans la sphère du sujet, elle reste difficile à appréhender dans les textes. Autant la critique se livre à une analyse précise des indices spatiotemporels du genre fictionnel, autant ses interprétations des textes lyriques restent abstraites. L'étude de certains exemples qualifiés d'équivoques montre les limites de ce modèle. Elle analyse notamment un poème circonstanciel de Brecht, "Wenn den Anstreicher durch die Lautsprecher über den Frieden redet » :

Schauen die Straßenarbeiter auf die Autostraßen

Und sehen

Knietiefen Beton, bestimmt für

Schwere Tanks.

Der Anstreicher redet vom Frieden.

Aufrichtend die schmerzenden Rücken

Die großen Hände auf Kanonenrohren

Hören die Gießer ihm zu.

Die Bombenflieger drosseln die Motoren

Und hören

Den Anstreicher vom Frieden reden.

Die Baumfäller stehen horchend in den stillen Wäldern

Die Bauern lassen die Pflüge stehn und halten die Hand hinters Ohr

Die Frauen bleiben stehn, die das Essen aufs Feld schleppen :

Auf dem umgebrochenen Acker steht ein Wagen mit Schalltrichtern. Von dort

Hört man den Anstreicher Frieden fordern. ${ }^{23}$

Pour conclure à la nature lyrique de l'énonciation, elle s'appuie sur la contradiction qui structure le poème, c'est-à-dire sur l'opposition entre les activités préparant la guerre et le discours sur la paix. Selon elle, cette forme est révélatrice de la relation spécifique entre le sujet et l'objet. Quelle est la nature de cette relation ? Peut-on la circonscrire en l'absence de définition du sujet lyrique? Pour Käte Hamburger, il n'est ni un «sujet théorique, ni un sujet historique, ni un sujet pragmatique ${ }^{24} »$. L'énonciation lyrique n'aurait d'autres fonctions qu'elle-même et exclurait les autres ${ }^{25}$. En l'occurrence, celle du poème de Brecht serait hors de la logique communicationnelle. Mais peut-on saisir la portée de ce poème sans l'inscrire dans son contexte et sans voir la référence à Hitler ? De même, peut-on dire que le sujet, dans les poèmes engagés d'Hugo, d'Aragon ou d'Éluard n'est pas lyrique parce qu'il est historique, c'est-à-dire parce que le sujet empirique est soumis à la situation? Ils paraissent indissociables. Pourtant, la charge émotionnelle reste intacte, indépendamment du contexte. Transgressant les frontières, le lyrisme peut s'adapter à toutes les situations de communication, de l'ancrage historique à la virtualité discursive la plus complète.

Si l'on cherche à analyser le genre lyrique en termes de pragmatique du langage, on se heurte de nouveau à un problème. Certes, sa valeur illocutoire - suivant la classification d'Austin et de Searle -, c'est-à-dire l'acte de parole accompli au moyen de l'énoncé lyrique, s'inscrit dans le domaine des actes expressifs. Mais quelle est sa valeur perlocutoire ? La comédie a pour but de faire rire pour corriger les mœurs, la tragédie de susciter la terreur et la pitié pour purger les passions, à quoi sert l'énoncé lyrique ? Faire ressentir, émouvoir, pourquoi?

Reste une autre piste à explorer, celle de la lecture du texte lyrique. Käte Hamburger affirme que «[n]otre expérience met en cause la seule énonciation lyrique du sujet énonciateur; il nous est interdit de dépasser les limites de son champ d'expérience où 
l'on peut dire qu'il nous tient captifs ${ }^{26} »$. Le texte lyrique tisserait donc une relation particulière avec le lecteur. Mais laquelle? Par exemple, Antonio Rodriguez formule l'hypothèse d'un pacte lyrique qui « articule la mise en forme du pâtir humain. Son effet global consiste à faire sentir et ressentir des rapports affectifs au monde ${ }^{27} »$. On comprend qu'il existe une structuration lyrique voulue par l'auteur pour induire «l'effet empathique du lyrisme ». Cependant, cette étude, comme d'autres, génère la frustration. Alors qu'il évoque un pacte, Antonio Rodriguez caractérise très peu l'impact de l'expression lyrique au niveau de la réception. Faire sentir, est-ce une fin en soi ? Pourquoi le lecteur lit-il un texte lyrique ? Ce qui apparaît comme un trait constant, l'expression des sentiments humains et la fonction émotive, conduit à une impasse dans la mesure où elle tend à annihiler la particularité du genre lyrique. De fait, n'est-ce pas le propre de la littérature, et de l'art en général, d'exprimer et de faire ressentir des passions humaines?

Il ne s'agit pas de faire une énième critique des critiques - au demeurant fort stimulantes - mais de montrer les limites des réflexions théoriques sur le genre lyrique, aussi rigoureuses et argumentées soient-elles. Il est frappant de constater le constant décalage entre discours théoriques, modèles abstraits et réalisations scripturales. Chaque théorie renvoie à un modèle partial et partiel, qui ne peut informer l'ensemble de la textualité lyrique. Dès lors, il parait délicat de traiter le lyrisme comme un genre.

\section{Le lyrisme comme principe transgénérique}

Il faudrait reconsidérer le statut de genre du lyrisme et l'envisager comme un principe transgénérique. Dans Qu'est-ce qu'un genre littéraire?, J.-M. Schaeffer distingue deux régimes génériques: par exemplification et par transformation ${ }^{28}$. Ces deux processus paraissent incompatibles avec le fonctionnement du genre lyrique. Pour ce qui est de l'exemplification, nous pensons avoir montré qu'il était vain de chercher à isoler des propriétés partagées par toutes les occurrences lyriques. Non seulement ce modèle virtuel relève de l'utopie mais les variations diachroniques et synchroniques sont trop importantes. La plupart du temps, la constitution du corpus se fait de manière intuitive ou en s'appuyant sur des indices paratextuels. L'ensemble constitué, sauf à opérer un tri orienté, est hétérogène.

On pourrait penser que le lyrisme est soumis à la «modulation générique », deuxième régime défini par J.-M. Schaeffer. Pourtant, si ce dernier permet de saisir la spécificité d'une œuvre, elle ne permet pas de saisir les propriétés qui font qu'elle appartient à un genre donné. Dans le cas du lyrisme, ces modulations se multiplient à l'infini, se conjuguent et se contredisent parfois jusqu'à faire perdre la relation aux autres textes. Les facteurs de transformations sont très variés : contextualisation historique, culturelle, modulations auctoriales, thématiques, etc. Ce qui frappe lorsque l'on confronte un modèle théorique à un corpus donné, c'est la quantité d'exceptions et de termes pour les désigner: lyrisme impersonnel de Baudelaire ou de Mallarmé, lyrisme critique de Corbière ou Rimbaud, lyrisme du quotidien de Laforgue ou Guillevic... Que l'on se réfère à un métadiscours d'auteur ou au commentaire d'un critique, l'actualisation lyrique diffère souvent de sa formulation théorique. Le lyrisme semble cultiver la coexistence de termes antithétiques et surtout être le lieu de tension entre modèle et réalisation textuelle. Le rapport de verticalité entre genre et textes est donc mis en échec. 
29 Un autre facteur nous incite à revoir le statut de genre du lyrisme: il ne s'intègre au système global qu'au prix de manipulations et de distorsions fallacieuses. L'impossible modélisation de sa textualité fait du lyrisme un principe transgénérique. Conçue comme double geste d'hybridation et de refondation des genres, la transgénéricité ${ }^{9}$ permet de décrire le fonctionnement transversal du lyrisme: ce dernier informe les textes en transcendant les autres catégories. En effet, le lyrisme entretient avec les autres genres un rapport plus complexe qu'un simple rapport d'exclusion ou d'opposition: il les contamine, leur emprunte certaines caractéristiques sans jamais complètement fusionner avec eux. Parcourant les "petits genres", Patrice Soler met en évidence les « interférences ${ }^{30}$ » intertextuelles et intergénériques. On constate que la notion de lyrisme revient à plusieurs reprises sans qu'il ait de sphère de référence circonscrite : il apparait aussi bien dans l'élégie ou le cantique que dans le caprice ou la tragédie. En somme, les textes lyriques s'inscrivent dans une logique d'intersection avec les autres réalisations textuelles. C'est ce qui explique que la définition du genre lyrique s'effectue en creux. Le lyrique n'est pas l'épique mais il n'est pas dépourvu d'une dynamique narrative; il n'est pas le dramatique mais présente des structures dialogiques; il est subjectif mais tend à la dépersonnalisation; il comporte des thèmes communs avec les autres genres mais les traite de manière spécifique. De même que l'on peut rencontrer des textes lyriques au sein des textes dramatiques ou épiques, de même, des textes lyriques intègrent certains traits de ces derniers. Pour ce qui est de l'épique, on peut penser à l'emphase oratoire et à la dynamique des recueils d'Hugo ou de Saint-John Perse. Quant au dramatique, il suffit de mentionner les Nuits de Musset ou « l'Après-midi d'un faune » de Mallarmé pour comprendre que le lyrisme s'élabore à partir d'une textualité théoriquement étrangère à la sienne.

$30 \mathrm{Au}$ lieu de s'en distinguer nettement, le poème lyrique se nourrit des autres genres et, réciproquement, régit une partie de leur textualité. C'est le cas en particulier dans le domaine des affects. S'appuyant sur l'impression que génère le texte lyrique, certains sortent de la logique générique et parlent comme Georges Molinié ${ }^{31}$ de "dominante tonale ». Selon lui, le lyrisme correspondrait à la volonté de l'auteur de susciter une vive émotion sur le lecteur par une écriture proche du chant. Mais de quelles émotions parlet-on? Évoque-t-on des sentiments amoureux comme le laisse penser la conjonction récurrente entre poésie lyrique et poésie amoureuse ${ }^{32}$ ? Envisage-t-on l'expression du désespoir, du deuil ou de la mélancolie, c'est-à-dire l'élégie? Que dire d'un poème comme "la Mort du Loup» de Vigny? Est-il tragique ou lyrique? La gamme des émotions extériorisées par le lyrisme est extrêmement variée et recouvre d'autres modes d'expression littéraires similaires.

31 Dans cette fonction émotionnelle et expressive, le lyrisme rencontre notamment le genre dramatique. Si la tragédie classique avait explicitement une vocation moralisatrice, elle avait également pour but de susciter la terreur et la pitié du public. Tragique, le personnage de Phèdre prononce des tirades proches du lyrisme : elle livre ses tourments intimes à ses interlocuteurs et aux spectateurs. La seule différence entre le dramatique et le lyrique semble résider dans la fonction et le point d'émergence de l'émotion. Contrairement aux autres genres qui modélisent les passions suivant les principes rhétoriques $^{33}$, le lyrisme n'aurait d'autres fins que d'émouvoir pour bouleverser le lecteur. On pourrait ainsi distinguer deux facettes de cette rencontre générique : l'un consacré à la progression dramatique et la démonstration scénique ; l'autre à atteindre le spectateur d'un point de vue affectif. Par exemple, dans Ruy Blas, les monologues de la 
reine comporteraient deux versants. D'une part, en engageant le corps du personnage, ils motivent le discours du déchirement et de la solitude sur le plan de l'action. D'autre part, ils assurent l'extériorisation et la figuration des passions. En outre, le lyrisme serait du côté de l'énonciateur, de l'expression alors que le dramatique serait du côté du destinataire, de la réception. Mais peut-on ainsi segmenter la communication affective ? Ne peut-on voir dans le lyrisme une composante, un tremplin émotionnel aux autres genres? En d'autres termes, un principe littéraire qui les transcenderait? Cette hypothèse est renforcée par l'histoire du lyrisme: l'une de ses caractéristiques intrinsèques réside dans sa capacité d'intégrer d'autres catégories, de s'adapter.

Le fonctionnement transversal du lyrisme est confirmé par le rapport qu'entretient le texte à son modèle. Le texte lyrique opère toujours un décalage avec le modèle sousjacent. Il déroge à la norme et brouille les repères. Qu'on ne s'y trompe pas, il ne s'agit pas de réduire le genre lyrique au phénomène moderne de brouillage des genres mais d'explorer un fonctionnement particulier. Dans un article ancien, Karlheinz Stierle écrit que la poésie lyrique «n'est pas un genre mais une manière propre de transgresser un schème générique ${ }^{34} »$. Selon lui, en l'absence de schème discursif de référence et à cause de la «multiplication des contextes simultanés » (c'est-à-dire de la polysémie inhérente à la construction figurale), l'énoncé lyrique tend toujours à la négation du discours. Pour le dire autrement, la coexistence de plusieurs modèles anéantit la possibilité d'une référence et remet en cause la linéarité du texte. On peut extrapoler cette réflexion. Dépassant les frontières génériques, le lyrisme instaure une perpétuelle tension entre modèle et réalisation, singularité et généralité, diachronie et synchronie. Mais plutôt que de voir dans ce mode de fonctionnement une menace pour l'intégrité du discours, ne peut-on y voir un principe qui accroit les possibles textuels?

De nombreux poèmes lyriques transmettent l'émotion à partir d'une remise en question des conditions de son expression. Cette réflexivité témoigne de la transgénéricité du lyrisme qui se nourrit du conflit entre textualité et modélisation. Un des exemples les plus significatifs se trouve au seuil des Amours jaunes, dans le poème intitulé « Ça » :

Des essais ? - Allons donc, je n'ai pas essayé !

Étude? - Fainéant je n'ai jamais pillé.

Volume ? - Trop broché pour être relié...

De la copie ? - Hélas non, ce n'est pas payé !

Un poème? - Merci, mais j'ai lavé ma lyre.

Un livre ? -... Un livre, encor, est une chose à lire !...

Des papiers? - Non, non, Dieu merci, c'est cousu!

Album? - Ce n'est pas blanc, et c'est trop décousu.

Bouts-rimés ? - Par quel bout ?... Et ce n'est pas joli !

Un ouvrage? - Ce n'est poli ni repoli.

Chansons? - Je voudrais bien, ô ma petite Muse !...

Passe-temps ? - Vous croyez, alors, que ça m'amuse ? [...]

... ÇA c'est naïvement une impudente pose ;

C'est, ou ce n'est pas ça : rien ou quelque chose...

- Un chef-d'œuvre? - Il se peut : je n'en ai jamais fait.

- Mais, est-ce du huron, du Gagne, ou du Musset?

- C'est du... mais j'ai mis là mon humble nom d'auteur,

Et mon enfant n'a pas même un titre menteur.

C'est un coup de raccroc, juste ou faux, par hasard...

L'Art ne me connaît pas. Je ne connais pas l'Art ${ }^{35 .}$

34 L'énumération des désignations génériques témoigne de la dispersion taxinomique, régissant le recueil. La répétition litanique de la question suivie d'une réponse négative 
montre que le lyrisme refuse toute classification. Pourtant, la dérision est loin d'effacer l'expression des sentiments. Malgré l'ironie, le lecteur sent l'intensité émotionnelle qui se noue autour de l'interrogation générique et de ses enjeux, notamment pour ce qui concerne l'identité du sujet.

Certes, en transgressant les règles de la communication et les repères littéraires, les textes lyriques mettent en danger le lien entre l'énonciateur et le récepteur de l'énoncé. Mais ils le renouvellent en le complexifiant: ils génèrent le lieu d'une rencontre singulière entre les deux pôles. Si l'on considère que le lyrisme crée des tensions et joue de l'écart entre l'expression et la réception, il faut que le lecteur reconnaisse cet écart et cette transgression. Le lecteur de textes lyriques ne se contente pas de s'émouvoir mais il doit être capable de mesurer la distorsion communicationnelle entre l'énonciateur et son destinataire, entre le langage et le monde évoqué, entre le texte et ses hypertextes. Ce lecteur critique doit être capable de tirer sens et jouissance des transgressions lyriques: de l'humour de Voiture lorsqu'il parle du "Rondeau d'Isabeau», de la provocation de Baudelaire dans "Une Charogne " ou de l'ironie rimbaldienne dans Une saison en enfer. Contrairement aux œuvres épiques et dramatiques qui usent des émotions pour agir directement sur le récepteur, les textes lyriques connaissent une utilisation double et indirecte des affects. Le lyrisme n'est pas seulement expression émotionnelle, mais aussi réflexion émotionnelle dans les deux sens du terme : image de l'émotion qui se donne à voir dans le texte et pensée sur la fonction de l'émotion dans la littérature. On assiste donc à un dédoublement de la textualité lyrique qui réalise dans le même temps son texte et son métatexte. Chaque œuvre lyrique génère son propre modèle et élabore sa textualité en traversant l'ensemble du champ littéraire et non par référence à un champ générique spécifique.

Influencé par un environnement théorique partial et des modèles variés, le lecteur s'attend à trouver une espèce de textes donnée. Cependant, cette attente, déçue par l'œuvre lyrique, l'oblige à élaborer un nouveau schéma à partir du texte singulier qu'il découvre. Le lecteur ne ressent pas pour ressentir, il se fait à son tour créateur dans l'intervalle que creuse l'œuvre lyrique entre expression et réception, texte et modèle. Loin de figer cette relation et la textualité qu'elle engage dans une autoréférentialité sclérosante, la transgénéricité lyrique ouvre le champ des possibles littéraires. Dès lors, nous ne sommes plus face à un fonctionnement générique mais face à un processus de création transversale qui engage une relation particulière entre les différents textes ainsi qu'entre le lecteur et le texte. On peut dire que la transgénéricité constitue une composante essentielle du lyrisme. D'une part, le texte lyrique n'a cessé de se nourrir des autres genres et des autres arts comme la musique et la peinture. D'autre part, par ce geste d'hybridation, il contient sa propre dynamique critique, redessinant perpétuellement ses contours théoriques.

37 À défaut de nourrir un modèle générique, le lyrisme nourrit la littérature critique. En tout cas, la distorsion entre modèle et textualité montre que le lyrisme n'obéit pas à la logique des autres genres. Contrairement à l'épique et au dramatique qui tissent une relation verticale entre textes et modèles, le lyrique opère toujours un décalage. C'est pour cette raison que l'on peut le qualifier de principe transgénérique : il se nourrit de la textualité des autres genres pour construire un modèle en perpétuel devenir. Paradoxalement, la transversalité et la rencontre avec des modèles extérieurs lui confèrent une dimension réflexive. Dépourvu de repères stables, le lecteur doit construire dans le même temps le texte et son métatexte. Cette relation singulière fonde l'originalité 
du lyrisme qui modifie les axes communicationnels, les frontières génériques et les discours théoriques. L'approche transgénérique ne doit pas s'appliquer uniquement à la littérature moderne. Elle permet de sortir du système générique traditionnel pour saisir la particularité du lyrisme, qui, défiant les classifications et les traditions, tire sa richesse de son impossible modélisation.

\section{BIBLIOGRAPHIE}

ARAGON, L. (1975 (1942)) : Les yeux d'Elsa, Paris, Seghers.

ARISTOTE (1991) : Rhétorique, texte établi et trad. par Médéric Dufour, Paris, les Belles lettres.

BAUDELAIRE, C. (1980 (1857)) : Les Fleurs du Mal, Paris, Laffont, « Bouquins ».

BRECHT, B. (1961 (1939)): Deutsche Kriegsfibel, in Svendenborger Gedichte, Berlin, Suhrkamp Verlag. BRODA, M. (1997) : L'amour du nom : essai sur le lyrisme et la lyrique amoureuse, Paris, Corti.

COMBE, D. (1989) : Poésie et récit : une rhétorique des genres, Paris, Corti.

CORBIÈRE, T. (1992) : Les Amours jaunes, Paris, Seuil.

FURETIÈRE, A. (2003) : « Poème » in Dictionnaire universel, 1690, Classique Garnier Numérique, http://www.classiques-garnier.com/numerique-bases/index.php?

module=App\&action=FrameMain.

GENETTE, G. (2004) : Fiction et diction, précédé d'Introduction à l'architexte, Paris, Seuil.

GUILLEVIC, E. (1989) : Art poétique, Paris, Gallimard.

HAMBURGER, K. (1986) : Die Logik der Dichtung, 1957, Logique des genres littéraires, traduit de l'allemand par Pierre Cadiot, Paris, Seuil.

HEGEL, G. W. F. (1997) : Esthétique, TII, Paris, Livre de Poche.

HUGO, V. (1972) : Les Contemplations, Paris, Livre de Poche.

MALLARMÉ, S. (1986) : Crise de vers, in Euvres complètes, Paris, Gallimard, « La Pléiade », 1945.

MOLINIÉ, G. (1986) : Éléments de stylistique française, Paris, Presses universitaires de France.

MONCOND'HUY, D.\& S CEPI, H. (ouvrage collectif sous la direction de) (2007) : Les genres de travers. Littérature et transgénéricité, Presses Universitaires de Rennes.

NIETZSCHE, F. (1994) : La Naissance de la tragédie, Paris, Livre de Poche.

RABATÉ, D. (ouvrage collectif sous la direction de) (1996) : Figures du sujet lyrique, Paris, Presses universitaires de France.

RODRIGUEZ, A. (2003) : Le Pacte Lyrique. Configuration discursive et interaction affective, Sprimont (Belgique), Pierre Mardaga éditeur.

RONSARD, P. DE (2001) : Les Quatre premier livres des Odes (1550), Le cinquiesme livre des Odes (1552), Odes (1547-1551), texte établi par Paul Laumonnier, Paris, Société des textes français modernes. 
SCHAEFFER, J.-M. (1989) : Qu'est-ce qu'un genre littéraire ?, Paris, Seuil. SÉDAR S ENGHOR, L. (1945) : Chants d'ombre, Paris, Seuil.

SOLER, P. (2001) : Genres, formes, tons, Paris, Presses Universitaires de France. STÉFAN, J. (1993 (1989)) : À la vieille Parque, Paris, Gallimard.

STIERLE, K. (1977) : « Identité du discours et transgression lyrique », Poétique n³2, Seuil, 1977, p. 431.

VALÉRY, P. (1930) : Littérature, in Morceaux choisis. Prose et poésie, Paris, Gallimard.

VILLON, F. (1991, (1533)) : Poésies complètes, Paris, Librairie Générale Française, Lettres Gothiques.

\section{NOTES}

1. Villon, 1991, (1533) : 311.

2. Stéfan, 1993, (1989).

3. Hugo, 1972 (1856)

4. Genette, 2004.

5. Ronsard, (1550-1552), $2001: 45$.

6. Ronsard : 48.

7. Ronsard : 37-41.

8. Ronsard : 222-224.

9. Furetière.

10. Valéry, $1930: 171$.

11. Combe, 1989.

12. Schaeffer, $1989: 177$.

13. Senghor, 1945.

14. Aragon, 1942, (1975) : 110.

15. Hegel, 1997 (1823) : 486. (16) Hugo, 1972 (1856) : 4.

16. Hugo, 1972 (1856) : 4.

17. Nietzsche, 1994 (1872) : 66.

18. Rabaté, 1996.

19. Mallarmé, 1945 (1897) : 366.

20. Guillevic, 1989.

21. Baudelaire, 1980(1857) : 68-69.

22. Hamburger, 1986 (1957) : 220.

23. Brecht, 1961, (1939) : 11-12.

24. Hamburger : 241.

25. Hamburger : 234.

26. Hamburger : 237.

27. Rodriguez, 2003 : 94.

28. Rodriguez : chapitre 4.

29. Moncond'huy et Scepi, $2007: 9$.

30. Soler, 2001.

31. Molinié, 1986.

32. Voir Broda, 1997.

33. Aristote, 1991.

34. Stierle, 1977 : 431.

35. Corbière, 1992 (1872) : 13-14. 


\section{RÉSUMÉS}

$\mathrm{Au}$ cœur des traditions critiques, les termes "lyrisme", "lyrique» semblent appliqués indifféremment à des textes présentant pourtant des différences énonciatives, stylistiques et formelles extrêmement importantes. L'hétérogénéité est telle qu'il semble difficile de sélectionner un modèle pour définir le genre lyrique. Contrairement au dramatique et à l'épique, il ne paraît pas être une catégorie issue de la convergence entre un modèle théorique et un ensemble des textes similaires. Le lyrisme semble échapper à un tel régime et s'inscrire davantage dans une logique transversale que dans une logique verticale. Le double mouvement, inductif et déductif, des textes aux modèles et des modèles aux textes, conduit à l'échec dans la mesure où il renvoie toujours à une vision partiale et partielle tant d'un point de vue théorique que textuel. Notre hypothèse est que le lyrisme suit un autre fonctionnement et que l'on peut le définir comme principe transgénérique. En effet, il se nourrit de la textualité des autres genres pour construire un modèle en perpétuel devenir. Dépourvu de repères stables, le lecteur doit construire dans le même temps le texte et son métatexte. Cette relation singulière fonde l'originalité du lyrisme qui modifie les axes communicationnels, les frontières génériques et les discours théoriques. Loin de s'appliquer uniquement à la littérature moderne, l'approche transgénérique permet de sortir du système générique traditionnel pour saisir la particularité du lyrisme, qui, défiant les classifications et les traditions, tire sa richesse de son impossible modélisation.

\section{INDEX}

Mots-clés : lyrisme, impossible modélisation, transgression textuelle et métatextuelle, transgénéricité, vecteur émotionnel

\section{AUTEUR}

\section{SANDRA GLATIGNY}

CÉRÉDI, Université de Rouen 